\title{
Gesundheit als Pflicht - Krankheit als Schuld? Warum Eigenverantwortung und Solidarität zusammengehören
}

Wir leben heute in einer Gesellschaft, die die Gesundheit zum höchsten Gut erklärt und zugleich die Illusion nährt, dass es allein am einzelnen Menschen liegt, allein an seiner Willensstärke, diese Gesundheit auch herbeizuführen, wenn er sich nur richtig anstrengt. Nicht nur medizinische Ratgeber, sondern zunehmend auch Praxen und Kliniken lassen Gesundheit als etwas erscheinen, das man mit genügend Mühe und Investition auch garantiert erreichen kann, das heißt als eine planbare Leistung.

Der gesunde Körper wird als Zeichen dafür gesehen, dass man hart genug an sich gearbeitet hat. Umgekehrt stellt sich das Krankwerden dann als etwas dar, was sich aus mangelnder Gesundheitskompetenz im Sinne einer mangelnden Investition in die eigene Gesundheit ergibt.

Vor diesem Hintergrund glauben wir heute, die medizinischen Probleme durch den Rückgriff auf die Eigenverantwortung lösen zu können. Das moderne Verständnis von Sozialstaat setzt immer weniger auf die Versorgung, sondern vielmehr auf die Aktivierung der Bürger und damit auf das Konzept der Eigenverantwortung des Individuums. Prämisse der Politik ist die Förderung der eigenen Kompetenzen des Bürgers, letztlich mit der Zielsetzung, ihn dadurch zur Übernahme eigener Verantwortung zu verpflichten. Man sagt zwar, dass man den Sozialstaat unbedingt erhalten wolle, aber de facto fährt man ihn zurück, und dies maskiert hinter akzeptanzverschaffenden Begriffen wie Freiheit, Wahlfrei- heit, Mündigkeit. Menschen sollen also vom Staat mit allen Voraussetzungen individuellen Erfolgs ausgestattet werden, damit sie anschließend in die selbständige Eigenverantwortung entlassen werden können. Und das soll in der Weise geschehen, dass man ihnen Emanzipation verspricht und zugleich Pflichten einfordert. Man könnte das auch unter dem Schlagwort „Fördern und Fordern“ subsumieren.

\section{Gesundheit ist in hohem Maße abhängig von strukturellen Rahmenbedingungen}

Natürlich ist es vernünftig, den mündigen Bürger und auch den mündigen Patienten anzustreben, aber es wird hier zu schnell und zu leicht übersehen, dass die Übernahme von Verantwortung an Grundvoraussetzungen geknüpft werden muss. $\mathrm{Zu}$ einseitig wird heute der Gesundheitszustand vornehmlich als individuelles Geschehen interpretiert, das geradezu ausschließlich abhängig ist von persönli-

\section{Der Autor}

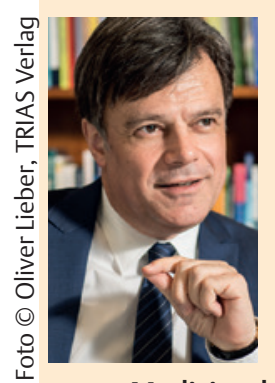

Professor Giovanni Maio war nach Medizin- und Philosophiestudium als Internist tätig. Seit 2005 hat er den Lehrstuhl für Medizinethik an der Albert-Ludwigs-Universität Freiburg und leitet dort das Institut für Ethik und Geschichte der Medizin. Er kritisiert die Machbarkeitsvorstellungen einer technisierten Medizin und tritt für eine neue Ethik der Besonnenheit ein.

Soeben ist Professor Maios neues Buch erschie-

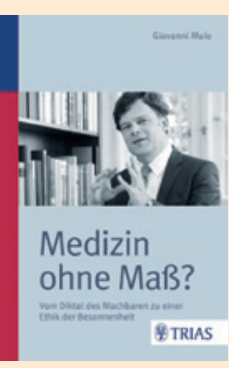
nen: „Medizin ohne Maß? Vom Diktat des Machbaren zu einer Ethik der Besonnenheit" (TRIAS Verlag). Darin thematisiert er Fragen, denen sich nicht nur Ärzte und Patienten dringend stellen sollten: Inwieweit ist Gesundheit machbar - und inwieweit auch Geschenk? Wohin führen uns die Versprechen der Reproduktionsmedizin? Verspricht das „schöner, besser, leistungsfähiger“ größeres Glück? Warum ist die Frage nach der Organspende schwieriger als uns suggeriert wird? Hat das Altsein nicht einen eigenen Wert? Und wie können wir eine Einstellung zum Sterben gewinnen, durch die wir uns nicht ausgeliefert fühlen? chem Gesundheitsverhalten. Das ist aber eine irrige Annahme. Gesundheit ist nicht einfach ein individuelles Persönlichkeitsmerkmal, sondern Gesundheit ist in hohem Maße abhängig von strukturellen Rahmenbedingungen und nicht vom Einzelnen allein. Wir haben es hier zuweilen mit einer Verengung der Verantwortungsperspektive zu tun.

\section{Unterprivilegierte Gruppen verfügen über weniger Möglich- keiten als höhere Schichten}

Beim Begriff der Eigenverantwortung wird zu leicht vergessen, dass die Gruppen der Bevölkerung, die das größte Risiko tragen zu erkranken, auch im Durchschnitt die geringsten Möglichkeiten und Fähigkeiten haben, die Gesundheitsförderung in ihrem Verhalten zu berücksichtigen. Das hat damit zu tun, dass die unterprivilegierten Schichten über weniger Freiräume verfügen, um in der Wahl ihres Lebensstils sich gesundheitsfördernd $\mathrm{zu}$ verhalten. Durch die strukturellen Lebensbedingungen sind sie oft in einer Lebensweise gefangen, die vor allem milieubedingt ist und ihnen weniger Freiheiten einräumt als bei höheren Schichten. Dieser Zusammenhang stellt ein Grundproblem aller Prävention dar, weil alle Ansätze zur Prävention in aller Regel die Menschen zuerst erreichen, die der Prävention am wenigsten bedürfen. Die Betonung der Eigenverantwortung ist hier eine zu einseitige Strategie, denn diesen Menschen fehlt es nicht an Aufgeklärtheit oder gutem Willen, sondern an inneren Ressourcen und vor allem an günstigen strukturellen Bedingungen. Daher muss gerade die Medizin dafür plädieren, dass sich die Politik nicht einfach darauf zurückzieht, die Verantwortung für seine Gesundheit ausschließlich dem Individuum zu übertragen. Sie muss auch verhindern, dass die an sich richtige Betonung der Eigenverantwortlichkeit nicht allmählich zu einer sanktionsbewehrten Einforderung von Gesundheit mutiert. Es gilt zu bedenken, dass sich der Staat mit dieser einseitigen Konzentrierung auf Aktivierung und Eigenverantwortung insgeheim den klassischen Marktgesetzen unterwirft und eine 
marktwirtschaftliche Denkweise übernimmt, die gerade für kranke Menschen katastrophal sein kann.

\section{Jeder Mensch kann nur dann eigenverantwortlich handeln, wenn er sich getragen weiß}

Die größte Gefahr eines zu einseitigen Kults der Eigenverantwortung liegt somit darin, dass unsere Gesellschaft versucht sein könnte, unter Verweis auf die Verantwortung des Einzelnen eine soziale $\mathscr{\rho}^{\dot{0}}$ Errungenschaft aufzugeben, nämlich die $\frac{\mathbb{\pi}}{2}$ Errungenschaft der Solidarität. Denn॰ über das Konzept der Aktivierung und\& der Eigenverantwortung wird ein Idealo der kompetitiven Lebensführung gepriesen und der Wert sozialer Orientierungen tendenziell abgeschwächt. Ein zu einsei ${ }_{N}^{5}$ tiger Pathos der Eigenverantwortung könnte am Ende einmünden in eine Erosion des Gemeinsinns, in einen Zerfall dero kohäsiven Kräfte in unserer Gesellschaft, in einen Bruch des Gemeinschaftsgefühls aller Menschen untereinander. Jeder: Mensch kann nur dann eigenverantwort-ब lich handeln, wenn er sich getragen weiß: von der Verlässlichkeit der sozialen Bin-ס्ष dungen, wenn er um einen stabilen Be-ब zugsrahmen weiß. Der Kult der Eigenverantwortung lässt diesen gemeinwohlorientierten Bezugsrahmen immer brüchi ger werden und treibt viele Menschen in eine Atmosphäre der Bedrohung und der⿳亠丷厂 Angst, nämlich der Angst vor sozialer Käl-ত te. Angst und Bedrohung aber sind keine $\frac{\ulcorner}{\Phi}$ guten Grundlagen, um zu eigenverant $-\frac{\mathrm{O}}{\mathrm{O}}$ wortlichem Handeln zu motivieren. Des: wegen muss das Konzept der Aktivierung® und der Eigenverantwortung mit Augen- $\frac{E}{3}$ maß verfolgt werden. Aus guten Gründen folgt ärztliches Handeln von jeher dem Ideal des bedingungslos Helfenden. Ver suche, dieses Paradigma aufzuweichen, rütteln an den Grundfesten der Medizin als soziale Praxis. Daher wäre gerade inP unseren Zeiten die Medizin gut beraten, nicht zum Richter über den Patienten zuธ mutieren, sondern Anwalt und Helfer des Patienten zu bleiben.

Korrespondenz

Prof. Dr. med. Giovanni Maio, M.A. phil.

Lehrstuhl für Medizinethik

Institut für Ethik und Geschichte der Medizin

Stefan-Meier-Str. 26

79104 Freiburg

Fax: 0761/203-5032

E-Mail: maio@ethik.uni-freiburg.de

klinikarzt 2014; 43 (9): 392-393 